\title{
Coronary artery calcium scoring on non-gated, non-contrast chest computed tomography (CT) using wide-detector, high-pitch and fast gantry rotation: comparison with dedicated calcium scoring CT
}

\author{
Jae Min Shin, Tae Hoon Kim, Ji Young Kim, Chul Hwan Park^ \\ Department of Radiology and the Research Institute of Radiological Science, Gangnam Severance Hospital, Yonsei University College of Medicine, \\ Seoul, Republic of Korea \\ Contributions: (I) Conception and design: JM Shin, TH Kim, CH Park; (II) Administrative support: TH Kim, CH Park; (III) Provision of study \\ materials or patients: JM Shin, JY Kim, CH Park; (IV) Collection and assembly of data: JM Shin, JY Kim, CH Park; (V) Data analysis and \\ interpretation: JM Shin, CH Park; (VI) Manuscript writing: All authors; (VII) Final approval of manuscript: All authors. \\ Correspondence to: Chul Hwan Park. Department of Radiology and the Research Institute of Radiological Science, Gangnam Severance Hospital, \\ Yonsei University College of Medicine, Seoul, Republic of Korea. Email: park_chulhwan@yuhs.ac.
}

Background: Our study assessed the reliability of non-gated, non-contrast chest computed tomography (NCCT) (with high pitch, wide coverage, and fast gantry rotation time, reconstructed at various slice thicknesses), compared with the electrocardiography (ECG)-gated calcium scoring cardiac computed tomography (CaCT), for quantifying coronary artery calcification (CAC).

Methods: Patients aged $\geq 50$ years who required clinical NCCT were prospectively enrolled. All CT scans were performed with 256-detector rows; z-axis coverage, $8 \mathrm{~cm}$; pitch, 1.5; and gantry rotation time, $280 \mathrm{~ms}$ (table feed, $42.86 \mathrm{~cm} / \mathrm{s}$ ). NCCT was followed by ECG-gated CaCT. The NCCT images were reconstructed at 0.625-, 1.25-, and 2.5-mm slice intervals. The CAC score was calculated on four sets of CT images with a commercially available software using the Agatston method. The CAC scores were divided into four standard Agatston scoring categories (Agatston scores: 0, 1-100, 101-400, and >400). The inter-observer and intertechnique agreements were evaluated for the CAC scores.

Results: Twenty-six patients (M:F, 14:12; mean age, 66.04 6.97 years) were evaluated. Agatston scores showed near-perfect correlation between CaCT and NCCT for each slice thickness. On Bland-Altman analysis, the mean differences of Agatston scores between CaCT and NCCT (slice thicknesses: 0.625, 1.25, and $2.5 \mathrm{~mm}$ ) were $37.54,6.67$, and -41.04 , respectively. Inter-technique concordance was high for the four Agatston scoring categories with linear-weighted kappa values of 0.599, 0.609, and 0.597 for NCCT (slice thicknesses: $0.625,1.25$, and $2.5 \mathrm{~mm}$, respectively). NCCT with $1.25-\mathrm{mm}$ slice thickness showed the strongest correlation with $\mathrm{CaCT}$.

Conclusions: CAC quantification with NCCT using a wide detector, high pitch, and high temporal resolution scanning modes correlates very highly with ECG-gated CaCT, and 1.25-mm slice thickness NCCT images are more reliable than other NCCT images.

Keywords: Agatston score; calcium score; cardiac computed tomography (cardiac CT); chest computed tomography (chest CT); high pitch

Submitted Mar 18, 2020. Accepted for publication Aug 05, 2020.

doi: $10.21037 /$ jtd-20-1371

View this article at: http://dx.doi.org/10.21037/jtd-20-1371

$\wedge$ ORCID: 0000-0002-0004-9475. 


\section{Introduction}

Cardiovascular disease (CVD) is by far the leading cause of death worldwide, with more than 17.9 million deaths in 2015. This figure is expected to rise to more than 23.6 million annually by 2030 (1-3). In $2015,43.8 \%$ of deaths due to CVD in the United States were caused by coronary artery disease (CAD), and medical costs for CAD are expected to nearly double between 2015 and 2030 (3). Coronary artery calcification (CAC) occurs almost exclusively in atherosclerotic arteries, and the extent of atherosclerotic plaque formation is a well-known marker indicating the total burden of coronary atherosclerosis (4). Previous large-scale prospective studies have confirmed that $\mathrm{CAC}$ is a strong independent predictor of $\mathrm{CAD}$, adverse cardiovascular events, and all-cause mortality $(5,6)$.

Electrocardiography (ECG)-gated calcium scoring cardiac computed tomography $(\mathrm{CaCT})$ is the standard reference method for the detection and quantification of CAC. CAC screening with ECG-gated $\mathrm{CaCT}$ has been accepted as a method to identify seemingly asymptomatic people with a high-risk of adverse cardiovascular events and to ensure the primary prevention of CVD (7). Compared with $\mathrm{CaCT}$, non-gated, non-contrast chest computed tomography (NCCT) is used in cases presenting with additional clinical indications, such as lung cancer screening, pneumonia, chronic obstructive pulmonary disease, and pleural disease. Previous reports have showed that approximately 10.6 million chest CT scans are taken per year in the United States, compared with 0.5 million calcium scoring CT scans and 0.3 million cardiac CT $(\mathrm{CaCT})$ scans $(8,9)$.

As the detection of asymptomatic patients with significant CAC is expected to contribute to reducing the incidences of adverse cardiovascular events and medical costs, the need for immediate and reliable CAC screening with non-gated NCCT has recently increased (10). However, respiratory and cardiac motion artifacts, as well as partial volume effects, have been the main obstacles to calculating the CAC scores from routine NCCT images. Recent advances in multi-detector CT scanners with high-pitch acquisition modes and thin slices can significantly reduce these artifacts and allow the possibility of integrating CAC scoring into routine chest CT scans $(11,12)$.

This study assessed the effectiveness of NCCT scan with a wide detector, high pitch, and fast gantry rotation in the quantification of CAC score, compared with ECG-gated $\mathrm{CaCT}$, and to evaluate the concordance of CAC scores between NCCT and ECG-gated CaCT scans. We present the following article in accordance with the MDAR reporting checklist (available at http://dx.doi.org/10.21037/jtd-20-1371).

\section{Methods}

\section{Study population}

A total of 30 patients aged 50 years and older, who had been referred for NCCT scans for various reasons, were prospectively enrolled in this study from April 2018 to May 2019. Three patients were excluded from the study, as previous scans showed no CAC. One patient who showed no CAC on the first chest CT scan was also excluded from the study, and underwent no calcium scoring cardiac CT scan. Thus, 26 patients [ 14 men and 12 women; mean age, $66.04 \pm 6.97$ years; mean body mass index (BMI), $\left.23.80 \pm 2.52 \mathrm{~kg} / \mathrm{m}^{2}\right]$ were prospectively registered in this study. NCCT was performed for evaluating the response of lung cancer to chemoradiation therapy $(\mathrm{n}=6)$, diagnosis or follow-up of benign lung diseases $(n=6)$, postoperative follow-up after lung cancer or lung metastasis $(n=5)$, diagnosis or follow-up of lung metastasis $(n=5)$ and diagnosis or follow-up of incidental lung nodules $(n=4)$. This prospective study was conducted in accordance with the Declaration of Helsinki (as revised in 2013), and approved by the institutional review board of Gangnam Severance Hospital (Approval No. 3-2018-0047). Informed consent was taken from all the patients (Figure 1).

\section{CT protocols}

All CT scans were performed with a wide-coverage, 256-detector row CT scanner (Revolution CT, GE Healthcare, Milwaukee, WI, USA) and a gantry rotation time of $280 \mathrm{~ms}$. NCCT scans were performed with a pitch of 1.5 and a table feed of $42.86 \mathrm{~cm} / \mathrm{s}$. The patients underwent scanning in the supine position while holding their breath at the end of each inspiration. The imaging protocol consisted of non-gated NCCT, followed by $\mathrm{CaCT}$ in the same examination. The CT protocol and its parameters are summarized in Table 1. Non-gated NCCT images of the thorax from the lung apex to the adrenal glands were acquired at $120 \mathrm{kVp}$ voltage, $70-150 \mathrm{mAs}$ tube current, and a slice thickness of $0.625 \mathrm{~mm}$. During image acquisition, the images were reconstructed at $0.625-, 1.25$, and $2.5-\mathrm{mm}$ slice intervals. CaCT was performed with a prospective ECG-gated acquisition protocol and images were obtained from the carina to the cardiac apex at middle 
diastole ( $75 \%$ of the $\mathrm{R}-\mathrm{R}$ interval). The imaging parameters were as follows: voltage, $120 \mathrm{kVp}$; tube current, 20-50 mAs; and reconstruction slice thickness, $2.5 \mathrm{~mm}$ (Table 1).

All CT images were transferred to a Picture Archiving and Communication System (PACS) station (Centricity 2.0;

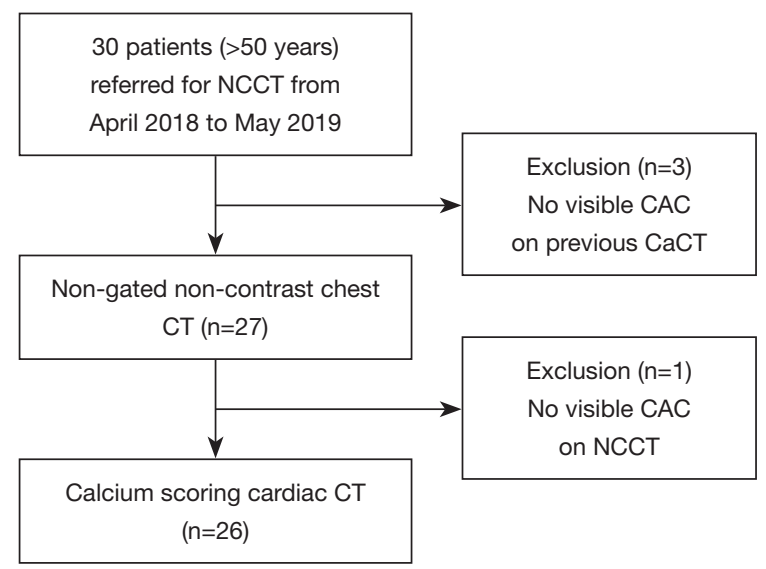

Figure 1 Flow chart of patient selection. NCCT, non-contrast chest computed tomography; CAC, coronary artery calcification; $\mathrm{CaCT}$, calcium scoring cardiac computed tomography.
GE Medical Systems, Mt Prospect, IL, USA). The axial images were transferred to a workstation for CAC scoring using a commercially available software (Aquarius iNtuition TM Ver.4.4.6, TeraRecon, Durham, NC, USA). The doselength-product (DLP) of NCCT and CaCT scans was systemically recorded.

\section{Image quality analysis}

The quality of CT images was evaluated by an observer (TH Kim, with >20 years of experience in cardiovascular radiology) from the axial NCCT images (slice thickness, $2.5 \mathrm{~mm}$ ), and from the CaCT images on the PACS station. According to the semi-quantitative, three-point grading scale, the image quality for calculating CAC scores was classified as poor (1, not suitable for CAC scoring due to cardiac motion artifacts), good (2, presence of cardiac motion artifacts but confident CAC scoring), or excellent (3, no artifacts affecting image quality are present).

\section{CAC scoring}

The CAC score was independently evaluated by two

Table 1 Chest and cardiac CT protocols for CAC quantification

\begin{tabular}{|c|c|c|}
\hline Parameters & Chest CT & Calcium scoring cardiac CT \\
\hline ECG-gating & Non-gated & Prospective ECG-gated, $75 \%$ of the R-R interval \\
\hline Detector coverage & Wide-coverage, 256-row (8 cm, z-axis) & Wide-coverage, 256-row (16 cm, z-axis) \\
\hline Tube rotation time (ms) & 280 & 280 \\
\hline Pitch & 1.5 (table feed: $42.86 \mathrm{~cm} / \mathrm{s}$ ) & - \\
\hline Tube voltage (kVp) & 120 & 120 \\
\hline Tube current (mAs) & $70-150$ & $20-50$ \\
\hline \multicolumn{3}{|l|}{ Reconstruction parameters } \\
\hline Matrix size & $512 \times 512$ & $512 \times 512$ \\
\hline FOV (mm) & $340.0-392.0$ & 170.0 \\
\hline Kernel & Standard kernel & Standard kernel \\
\hline Reconstruction algorism & Filtered back projection & Filtered back projection \\
\hline
\end{tabular}

$\mathrm{CT}$, computed tomography; CAC, coronary artery calcification; ECG, electrocardiography; kVp, peak kilovoltage; mAs, milliamperesecond; FOV, field of view; DLP, dose length product. 
Table 2 Patient characteristics and risk profiles

\begin{tabular}{|c|c|}
\hline Variables & Value \\
\hline Age (years) ${ }^{+}$ & $66.04 \pm 6.97$ \\
\hline \multicolumn{2}{|l|}{ Sex } \\
\hline Female & $12(46.15)$ \\
\hline Male & $14(53.85)$ \\
\hline BMI $\left(\mathrm{kg} / \mathrm{m}^{2}\right)^{+}$ & $23.80 \pm 2.52$ \\
\hline Heart rate $(/ \min )^{\star^{+}}$ & $70.81 \pm 12.78$ \\
\hline \multicolumn{2}{|l|}{ DM } \\
\hline No & 22 (84.62) \\
\hline Yes & $4(15.38)$ \\
\hline \multicolumn{2}{|l|}{ Hypertension } \\
\hline No & $16(61.54)$ \\
\hline Yes & $10(38.46)$ \\
\hline \multicolumn{2}{|l|}{ Hyperlipidemia } \\
\hline No & 22 (84.62) \\
\hline Yes & $4(15.38)$ \\
\hline \multicolumn{2}{|l|}{ CAD } \\
\hline No & 24 (92.31) \\
\hline Yes & $2(7.69)$ \\
\hline \multicolumn{2}{|l|}{ Smoking } \\
\hline No & 20 (76.92) \\
\hline Yes & $6(23.08)$ \\
\hline
\end{tabular}

Data are numbers of subjects with percentages in parentheses unless otherwise noted. ${ }^{+}$, data are presented as the mean \pm standard deviation; *, heart rates were recorded during the cardiac CT scans. BMI, body mass index; DM, diabetes mellitus; $\mathrm{CAD}$, coronary artery disease.

observers (TH Kim and CH Park, with >20 and >10 years of experience in cardiovascular radiology, respectively) using Aquarius (TeraRecon) software. Four sets of CT images, consisting of NCCT images (slice thicknesses: 0.625, 1.25 , and $2.5 \mathrm{~mm}$ ) and ECG-gated CaCT images, were presented to the observers in a random, blinded manner. The CAC score was estimated when the lesion showed a peak attenuation value of $>130$ Hounsfield units (HU) and a minimum area of $1.02 \mathrm{~mm}^{2}$ (at least 3 contiguous pixels). The lesion score was calculated using the Agatston method by multiplying the lesion area by the attenuation factor (derived from the maximum $\mathrm{HU}$ within the lesion) as follows: factor 1 for lesions with 130-199 HU, 2 for 200-
$299 \mathrm{HU}, 3$ for 300-399 HU, and 4 for $\geq 400 \mathrm{HU}$ (13).

The region of interest in each coronary artery was manually encircled and the lesion with CAC was automatically depicted. The area of the lesion and the CAC score were automatically measured. The total Agatston score of the CAC lesion was automatically calculated by summation of the scores of individual lesions with CAC. For CAD risk stratification, the Agatston scores of CAC lesions were further classified into the following four Agatston scoring categories: category 0, CAC score $0 ; 1$, $1-100 ; 2,101-400$; and 3, >400. These four categories have been used in several previous studies (14-16) and are also commonly used in clinical practice.

\section{Statistical analysis}

Categorical variables are presented as frequencies (in percentages). Continuous variables are presented as mean \pm standard deviation (SD) for normally distributed variables, and as median [interquartile range (IQR)] for non-normally distributed variables. Normality assumptions for continuous variables were confirmed with Shapiro-Wilk test and Kolmogorov-Smirnov test. The continuous variables were analyzed using Pearson's correlation. Inter-group comparisons were performed by two independent sample $t$-tests.

The inter-observer and inter-technique agreements on the quantitative CAC scores were evaluated using Bland-Altman analysis, intraclass correlation coefficient (ICC), kappa value, and variability. The concordance of the Agatston scoring categories with the four sets of CT images was expressed as weighted kappa values. ICCs or kappa values of $<0.20,0.21-0.40,0.41-0.60,0.61-0.80$, and 0.81-1.00 indicated slight, fair, moderate, substantial, and almost perfect agreement, respectively (17). A P value of 0.05 or less was considered a statistically significant difference. Statistical analyses were performed a using commercially available software (R package version 3.4.4, SAS Institute Inc., Cary, NC, USA).

\section{Results}

The physical characteristics and risk profiles of patients are summarized in Table 2. The presence of smoking history was associated with CAC scores $(\mathrm{P}=0.020)$. Other characteristics or risk factors did not significantly affect CAC scores $(\mathrm{P}>0.05)$. The mean DLP was $239.69 \pm$ $50.65 \mathrm{mGy} \cdot \mathrm{cm}(145.03-327.68 \mathrm{mGy} \cdot \mathrm{cm})$ for NCCT 

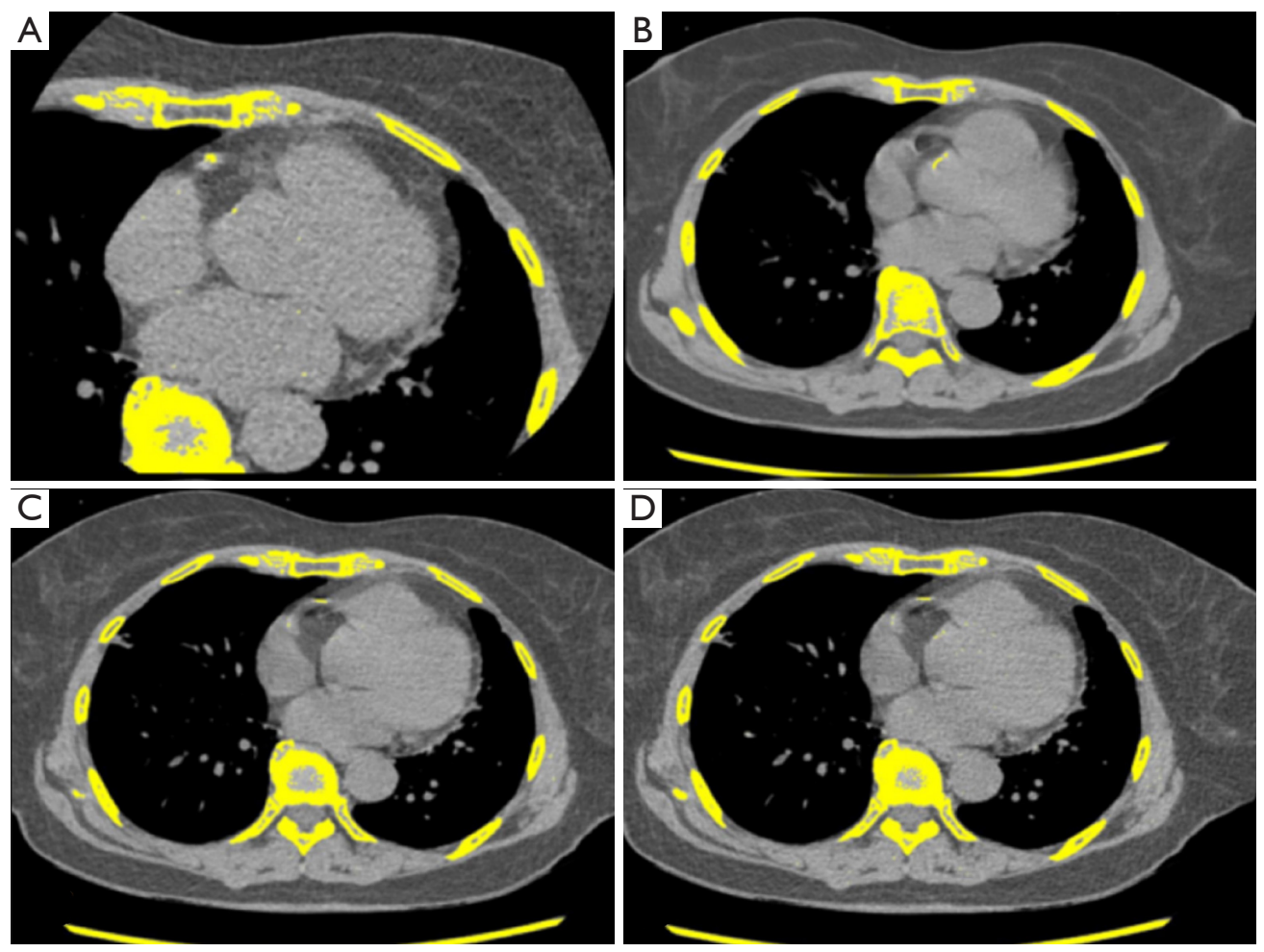

Figure 2 Comparison of visualized coronary artery calcification (CAC) among the calcium scoring cardiac computed tomography (CaCT) scan and the non-contrast chest computed tomography (NCCT) scans. (A) CaCT scan shows CAC on right coronary artery (RCA); CAC Agatston score is 33.40. (B) NCCT scan (slice thickness $2.5 \mathrm{~mm}$ ) shows a false-negative result of CAC. (C,D) NCCT scans (slice thickness 1.25 and $0.625 \mathrm{~mm}$, respectively) show CAC on RCA; CAC Agatston scores are 13.38 and 23.47, respectively.

acquisitions, and $26.92 \pm 9.07 \mathrm{mGy} \cdot \mathrm{cm}(14.66-55.49 \mathrm{mGy} \cdot \mathrm{cm})$ for $\mathrm{CaCT}$ acquisitions. The mean effective doses of NCCT and $\mathrm{CaCT}$ with a conversion factor of 0.017 were $4.07 \pm 0.86$ $\mathrm{mSv}$ and $0.46 \pm 0.15 \mathrm{mSv}$, respectively.

\section{Image quality of chest and cardiac CT scans}

Among the 26 patients, six axial NCCT images (6/26; $23.08 \%$ ) (slice thickness, $2.5 \mathrm{~mm}$ ) were graded with an image quality score of 3 , and 20 images (20/26; 76.92\%) has a score of 2 . For CaCT images, 18 images (18/26; $69.23 \%$ ) were graded with a score of 3 and eight images $(8 / 26 ; 30.77 \%)$ has a score of 2 . The mean heart rate during CaCT scans, patients' BMI, and DLP showed no significant differences between the grade 2 group and grade 3 image groups.

\section{CAC scores}

The CAC score was estimated for all patients using the
$\mathrm{CaCT}$ images. In one patient (Agatston score of 33.40 on the $\mathrm{CaCT}$ images), a false-negative result $(1 / 26 ; 3.85 \%)$ was registered on one NCCT image (slice thickness, 2.5 $\mathrm{mm}$ ), whereas lesions with CAC could be seen on the other NCCT images (slice thicknesses of 0.625 and $1.25 \mathrm{~mm}$ ) (Figure 2).

The median Agatston scores were 139.25 (IQR, 109.05309.38), 119.88 (IQR, 84.30-276.14), and 75.52 (IQR, 55.58-207.90) for NCCT images (slice thicknesses: 0.625, 1.25 , and $2.5 \mathrm{~mm}$, respectively), and 109.75 (IQR, 76.37207.90) for $\mathrm{CaCT}$ images. The inter-technique reliability for CAC scoring is summarized in Tables 3,4. The intertechnique agreement of Agatston scores between $\mathrm{CaCT}$ and NCCT scans was excellent at all slice thicknesses.

In contrast, the inter-technique agreement in the four Agatston scoring categories was substantial between CaCT and NCCT scans (slice thickness, $1.25 \mathrm{~mm}$ ) (linear-weighted kappa value of 0.609), and moderate in the scans with the other two slice thicknesses $(0.625$ and $2.5 \mathrm{~mm}$; linear-weighted kappa values of 0.599 and 0.597 , 
Table 3 Agreement of coronary artery calcium Agatston scores between each non-contrast chest computed tomography (NCCT) scan and the calcium scoring cardiac computed tomography (CaCT) scan

\begin{tabular}{lcc}
\hline $\begin{array}{l}\text { Slice thickness } \\
\text { of NCCT }\end{array}$ & ICC $(95 \% \mathrm{Cl})$ & Variability (\%) \\
\hline $0.625 \mathrm{~mm}$ & $0.8940(0.7592-0.9529)$ & 31.53 \\
$1.25 \mathrm{~mm}$ & $0.9167(0.8236-0.9617)$ & 27.43 \\
$2.5 \mathrm{~mm}$ & $0.8150(0.6120-0.9147)$ & 45.36 \\
\hline
\end{tabular}

ICC, intraclass correlation coefficient; $\mathrm{Cl}$, confidence interval.

Table 4 Agreement of Agatston scoring categories between each non-contrast chest computed tomography (NCCT) scan and the calcium scoring cardiac computed tomography (CaCT) scan

\begin{tabular}{lcc}
\hline $\begin{array}{l}\text { Slice thickness } \\
\text { of NCCT }\end{array}$ & $\begin{array}{c}\text { Linear weighted } \\
\text { kappa }(95 \% \mathrm{Cl})\end{array}$ & $\begin{array}{c}\text { Quadratic weighted } \\
\text { kappa }(95 \% \mathrm{Cl})\end{array}$ \\
\hline $0.625 \mathrm{~mm}$ & $0.599(0.331-0.867)$ & $0.676(0.436-0.917)$ \\
$1.25 \mathrm{~mm}$ & $0.609(0.340-0.897)$ & $0.692(0.457-0.926)$ \\
$2.5 \mathrm{~mm}$ & $0.597(0.373-0.821)$ & $0.710(0.535-0.884)$ \\
\hline
\end{tabular}

$\mathrm{Cl}$, confidence interval.

respectively). NCCT scan with a slice thickness of $1.25 \mathrm{~mm}$ had the highest ICC or weighted kappa value, and the least variability in both Agatston scores and Agatston scoring categories, showing the strongest correlation with $\mathrm{CaCT}$. This was followed by NCCT scan with a slice thickness of $0.625 \mathrm{~mm}$. Results of Bland-Altman analysis showed that the mean differences between CaCT and NCCT (slice thicknesses: $0.625,1.25$, and $2.5 \mathrm{~mm}$ ) were $37.54,6.67$, and -41.04 , respectively (Figure 3).

The ICCs for inter-observer reliability were excellent in both the Agatston score and the Agatston scoring categories for the four sets of CT images (Tables 5,6).

However, between the two readers, there were three discordant Agatston scoring categories in two patients. One discordant score was observed in the NCCT scan (slice thickness of $1.25 \mathrm{~mm}$ ) (86.47 vs. 104.27), while the other two discordant scores were observed in the CaCT scans (106.54 vs. 98.75 and 102.96 vs. 95.06). Table 7 shows the number of concordant and discordant results between the CaCT images and the three sets of NCCT images in the Agatston scoring categories. NCCT scans (slice thickness, $0.625 \mathrm{~mm}$ ) had seven discordant cases $(7 / 26 ; 26.92 \%)$ : score 1 was overestimated as score 2 in five patients $(5 / 26$; $19.23 \%)$ and the other two patients $(2 / 26 ; 7.69 \%)$ were underestimated by 1 category ( 2 instead of 1 and 3 instead of 2). In the NCCT scans (slice thickness, $1.25 \mathrm{~mm}$ ), 4 patients (4/26; $15.38 \%)$ were overestimated by 1 category ( 1 instead of 2 ) and 3 patients $(3 / 26 ; 11.54 \%)$ were underestimated by 1 category [ 2 instead of $1(n=2)$ and 3 instead of $2(n=1)]$. Therefore, the net reclassification rate was $26.92 \%(7 / 26)$. In the NCCT scans (slice thickness $2.5 \mathrm{~mm}$ ), all eight cases of discordance $(8 / 26 ; 30.77 \%)$ were underestimated by 1 category [ 1 instead of $0(n=1) ; 2$ instead of $1(n=5) ; 3$ instead of $2(\mathrm{n}=2)$ ] (Figures 4,5$)$.

The mean age, BMI, and DLP were not significantly different between the patients with concordant and discordant scoring categories. However, the mean heart rate measured during $\mathrm{CaCT}$ was significantly higher in patients with discordant score categories than that in patients with concordant scoring categories $(74.53 / \mathrm{min} v s .65 .73 / \mathrm{min}$, respectively; $\mathrm{P}<0.001)$.

\section{Discussion}

This study evaluated the reliability of NCCT images using a wide detector, fast gantry rotation, and a high-pitch scanning mode for quantifying CAC scores, and compared those with the scores estimated from ECG-gated calcium scoring $\mathrm{CaCT}$ scans. Our study demonstrated that the agreement of Agatston scores was excellent between the ECG-gated CaCT and NCCT scans at each slice thickness. The agreement on the four Agatston scoring categories was strong between CaCT and NCCT (slice thickness, $1.25 \mathrm{~mm}$ ) and was moderate between $\mathrm{CaCT}$ and the remaining two slice thicknesses of NCCT. NCCT (slice thickness, $1.25 \mathrm{~mm}$ ) had the highest ICC or weighted kappa values, and the least variability in the Agatston scores and Agatston scoring categories, thus showing the strongest correlation with $\mathrm{CaCT}$.

Many recent studies have suggested that CAC screening in the asymptomatic population could greatly help to prevent the incidence of adverse cardiovascular events $(6,18-22)$. Chest CT scans are more likely to be used as a CAC screening tool because a larger proportion of the asymptomatic population undergo chest $\mathrm{CT}$ than $\mathrm{CaCT}$ CT scans, and also because of the improvements in the temporal resolution of CT systems. In addition, recent studies comparing automated CAC scoring in either ECGgated coronary CT or non-gated chest CT with standard manual assessment reported that automated CAC scoring is feasible with acceptable agreement and reliability (23-25). Automated CAC scoring may save time and labor, 

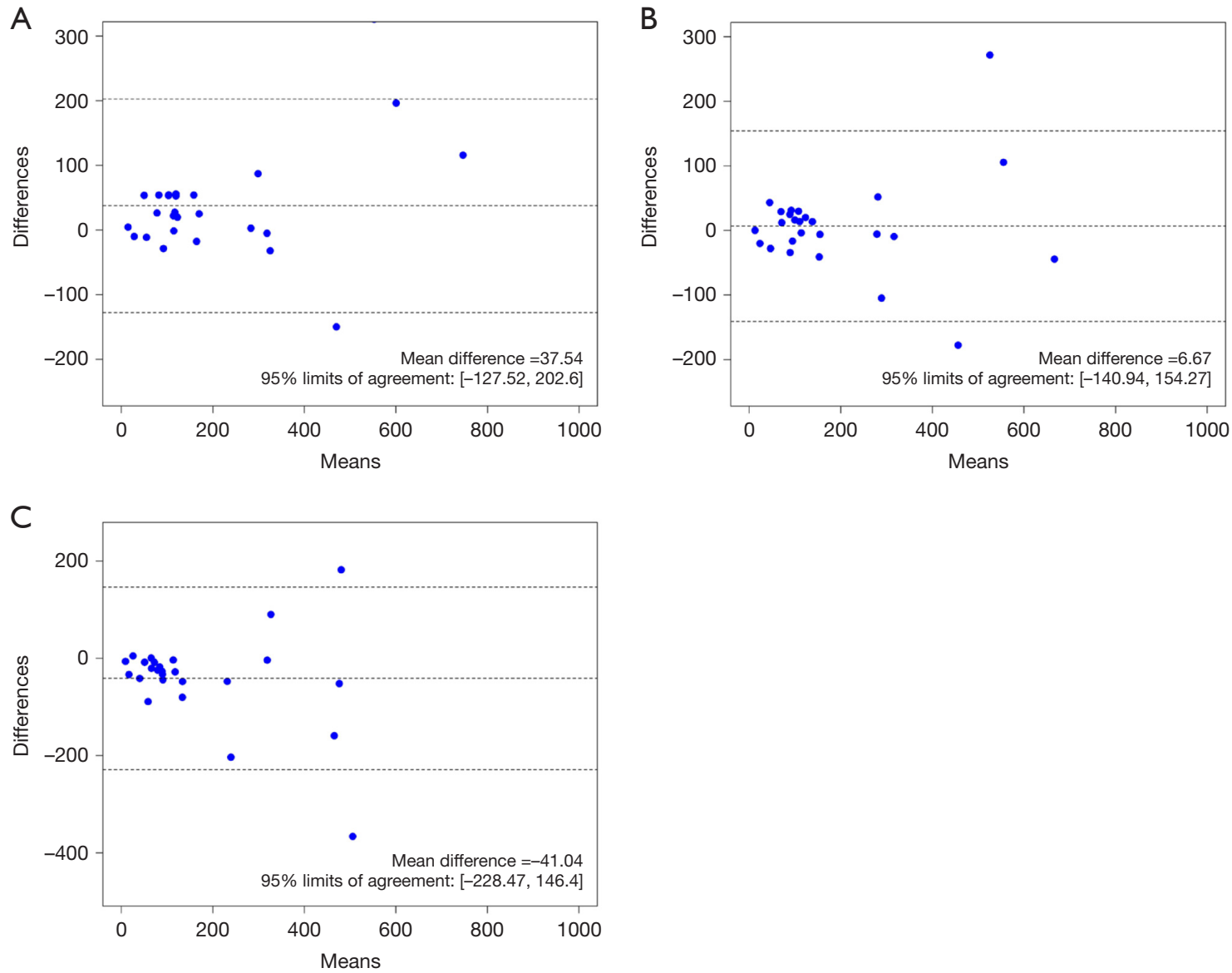

Figure 3 Bland-Altman plots demonstrating the comparison of coronary artery calcification (CAC) Agatston scores between each noncontrast chest computed tomography (NCCT) scan and calcium scoring cardiac computed tomography (CaCT) scan. (A) The mean difference in Agatston scores between NCCT (slice thickness $0.625 \mathrm{~mm}$ ) and CaCT is 37.54 [95\% confidence interval (CI): -127.52 to 202.60]. (B) The mean difference in Agatston scores between NCCT (slice thickness $1.25 \mathrm{~mm}$ ) and CaCT is 6.67 (95\% CI: -140.94 to 154.27). (C) The mean difference in Agatston scores between NCCT (slice thickness $2.5 \mathrm{~mm}$ ) and CaCT is 41.04 (95\% CI: -228.47 to 146.40).

Table 5 Inter-observer agreement in Agatston scores for the four sets of CT scans

\begin{tabular}{lcc}
\hline Acquired CT scan & ICC $(95 \% \mathrm{Cl})$ & Variability (\%) \\
\hline NCCT $(0.625 \mathrm{~mm})$ & $0.9992(0.9976-0.9997)$ & 2.25 \\
NCCT $(1.25 \mathrm{~mm})$ & $0.9952(0.9895-0.9979)$ & 2.81 \\
NCCT $(2.5 \mathrm{~mm})$ & $0.9996(0.9991-0.9998)$ & 1.17 \\
CaCT & $0.9997(0.9992-0.9999)$ & 1.64 \\
\hline
\end{tabular}

The slice thicknesses of the chest CT scans are presented in parentheses, after NCCT. ICC, intraclass correlation coefficient; NCCT, non-contrast chest computed tomography; CaCT, calcium scoring cardiac computed tomography; $\mathrm{Cl}$, confidence interval.
Table 6 Inter-observer agreement in Agatston scoring categories for the four sets of CT scans

\begin{tabular}{lcc}
\hline Acquired CT scan & $\begin{array}{c}\text { Linear weighted } \\
\text { kappa }(95 \% \mathrm{Cl})\end{array}$ & $\begin{array}{c}\text { Quadratic weighted } \\
\text { kappa }(95 \% \mathrm{Cl})\end{array}$ \\
\hline NCCT $(0.625 \mathrm{~mm})$ & $1.000(1.000-1.000)$ & $1.000(1.000-1.000)$ \\
NCCT $(1.25 \mathrm{~mm})$ & $0.938(0.816-1.000)$ & $0.949(0.847-1.000)$ \\
NCCT $(2.5 \mathrm{~mm})$ & $1.000(1.000-1.000)$ & $1.000(1.000-1.000)$ \\
CaCT & $0.841(0.670-1.000)$ & $0.879(0.745-1.000)$ \\
\hline
\end{tabular}

The slice thicknesses of the chest CT scans are presented in parentheses. NCCT, non-contrast chest computed tomography; $\mathrm{CaCT}$, calcium scoring cardiac computed tomography; $\mathrm{Cl}$, confidence interval. 
Table 7 Concordant and discordant results between calcium scoring cardiac computed tomography (CaCT) and each of the three sets of noncontrast chest computed tomography (NCCT) images in Agatston scoring categories

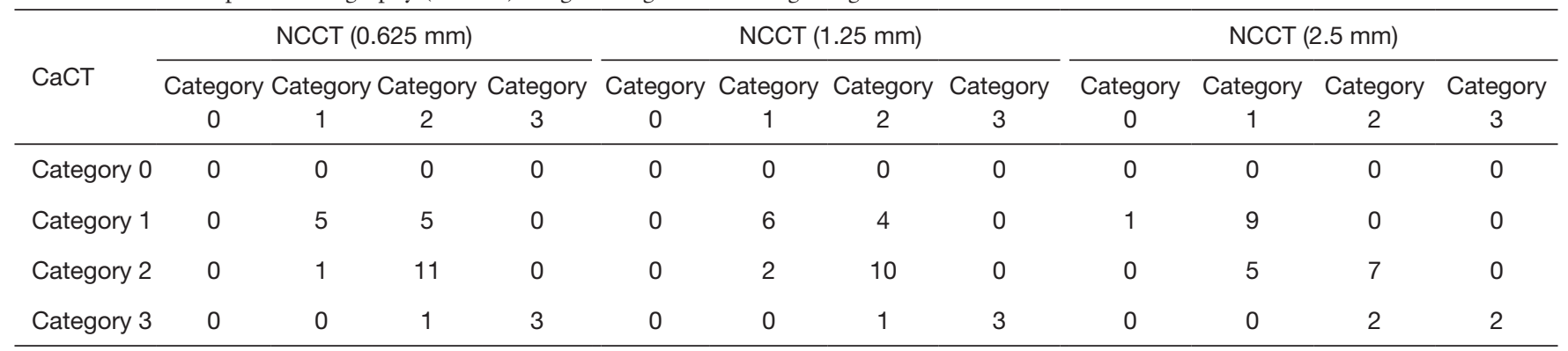
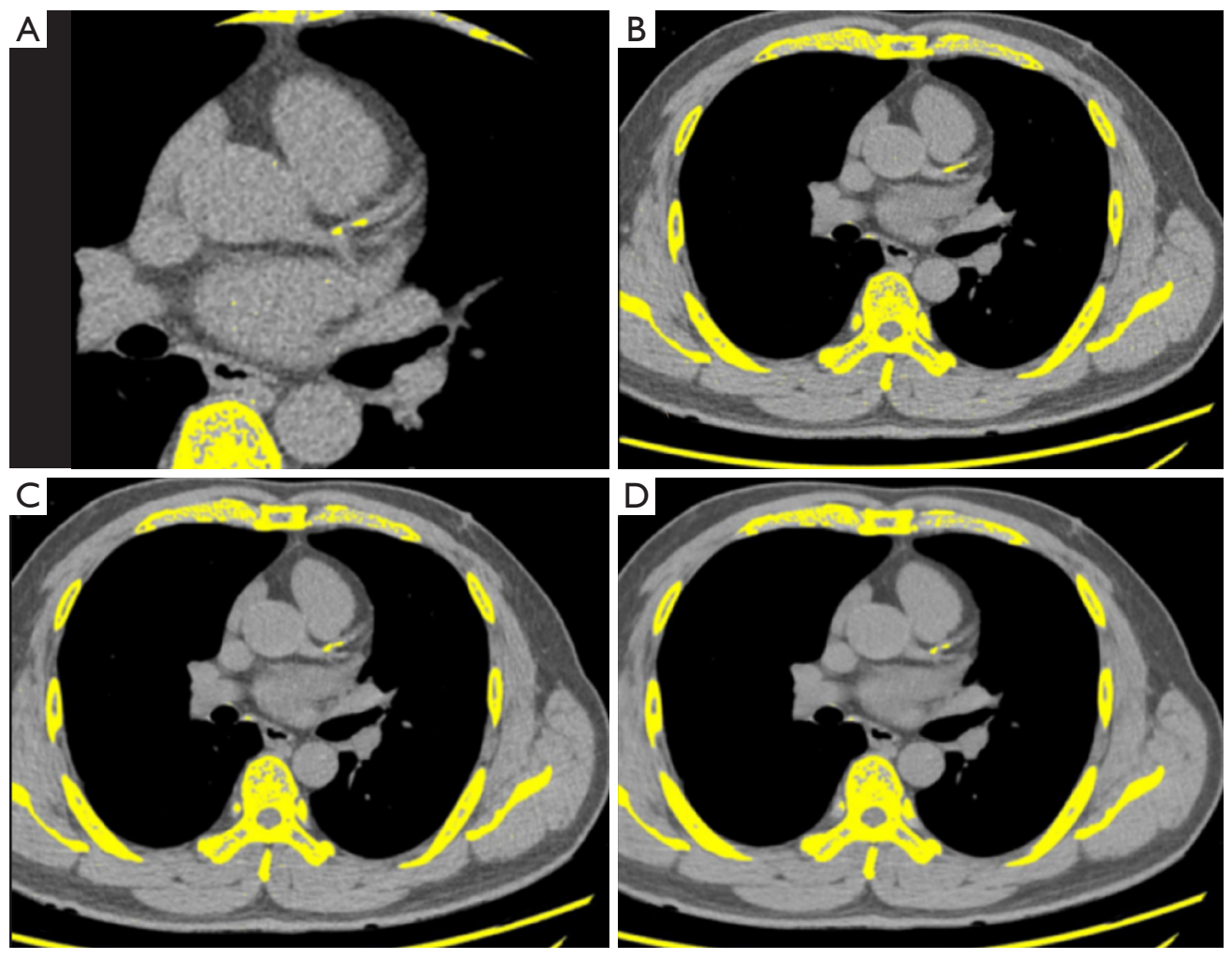

Figure 4 Overestimation of Agatston scores in the non-contrast chest computed tomography (NCCT) scan (slice thickness 0.625 and $1.25 \mathrm{~mm}$ ), compared with the calcium scoring cardiac computed tomography (CaCT) scan. (A) A 70-year-old man has coronary artery calcification (CAC) on the left anterior descending artery (LAD). (B,C,D) CAC Agatston scores in CaCT and NCCT (slice thicknesses 0.625, 1.25 , and $2.5 \mathrm{~mm}$ ) are 55.02, 109.05, 84.30, and 47.05, respectively; Agatston scoring categories are 1, 2, 1, and 1, respectively.

making CAC scoring possible in routine chest CT scans. One of the strategies for the use of a chest CT scan for CAC screening may be to use the widest detector coverage, the highest pitch and the fastest rotation time permitted by the CT system. Our preliminary results show that reliable CAC scores can be obtained from non-gated chest CT scans with a $\mathrm{z}$-axis coverage of $8 \mathrm{~cm}$, pitch of 1.5 , and a gantry rotation time of $280 \mathrm{~ms}$. This scanning mode covers $42.86 \mathrm{~cm} / 1 \mathrm{~s}$, which could reduce the motion artifact due to a beating heart. In our study, however, the CAC scores were overestimated in the NCCT images (slice thicknesses of 0.625 and $1.25 \mathrm{~mm}$ ) (median, 139.25 and 119.88) as compared with the CaCT images (109.75). The difference in CAC scores was much greater in the NCCT scan with a 

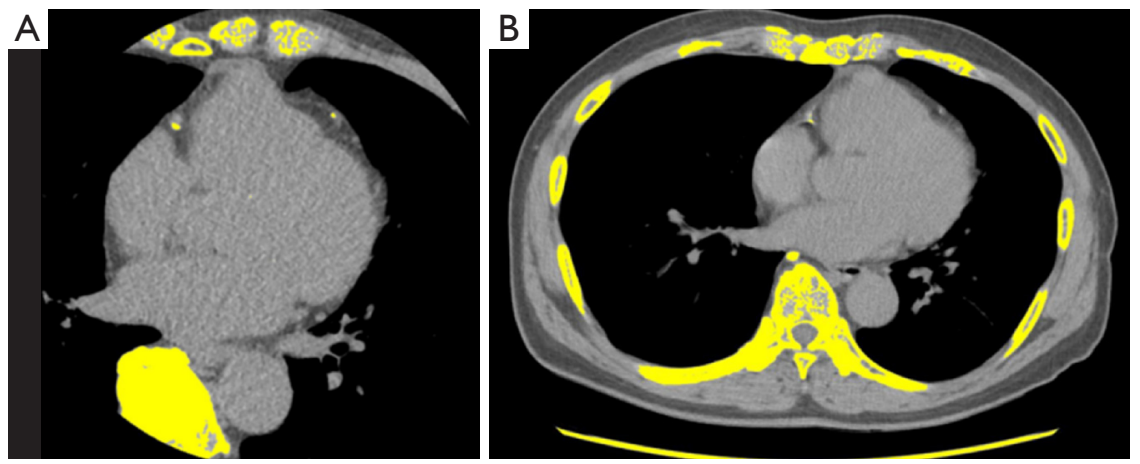

Figure 5 Underestimation of Agatston score in the non-contrast chest computed tomography (NCCT) scan (slice thickness 2.5 mm), compared with the calcium scoring cardiac computed tomography (CaCT) scan. A 68-year-old woman has coronary artery calcification (CAC) on the left anterior descending artery and right coronary artery. CAC Agatston scores in CaCT and NCCT (slice thickness 2.5 mm) are 112.95 and 68.72, respectively; Agatston scoring categories are 2 and 1, respectively.

slice thickness of $0.625 \mathrm{~mm}$ (mean difference, 37.54) than in those with slice thickness of $1.25 \mathrm{~mm}$ (mean difference, 6.67). This overestimation is likely to have included systolic and diastolic images from the non-gated chest CT scan, and coronary artery motion artifacts are common in the systolic cardiac phase. Previous studies have mentioned overestimation of size due to blurred images from motion artifacts $(18,26)$. Another potential reason for this overestimation may be that the partial volume effect in the thinner slice thickness of chest CT has decreased, increasing the attenuation of the coronary calcification (26). In our study, the CAC score increased in the NCCT with slice thicknesses of 0.625 and $1.25 \mathrm{~mm}$. For NCCT images with a slice thickness of $1.25 \mathrm{~mm}$, however, the CAC score was close to that of CaCT images. This is thought to be caused by the thin slice thickness, which reduces partial volume effects, and blurs the images during NCCT as compared to the ECG-gated CaCT, at a slice thickness of $2.5 \mathrm{~mm}$ (26).

In contrast, NCCT (slice thickness, $2.5 \mathrm{~mm}$ ) underestimated the CAC scores (median, 75.52) and had one false-negative CAC. Non-gated CT scans may miss small lesions with CAC due to motion artifacts $(19-21,26)$. In addition, the thicker the slice intervals, the more the chances of lesions being missed. A potential explanation for this is that thick slices reduce blooming artifacts on NCCT images as compared with ECG-gated CaCT images by increasing the attenuation and area of the calcified lesions $(21,26)$. For CAC scoring, false-positive results are considered to occur with high image noise. In our study, there were no false-positive results, suggesting that all 26 of our image sets maintained acceptable image quality for

\section{CAC scoring $(21,26)$.}

Heart rate may be an important factor in the discordant results of the Agatston scoring categories. In our study, the mean heart rate measured during cardiac CT was much higher in patients with discordant scoring categories than in patients with concordant scoring categories $(74.53 / \mathrm{min}$ vs. $65.73 / \mathrm{min}, \mathrm{P}<0.001)$. However, chest $\mathrm{CT}$ is usually performed without ECG-gating; therefore, it is difficult to accurately assess the discordant effects of heart rate. In this study, NCCT images with slice thickness of $0.625 \mathrm{~mm}$ overestimated the Agatston scoring categories (5/7; 71.43\%), whereas those with slice thickness of $2.5 \mathrm{~mm}$ underestimated the Agatston scoring categories (8/8; $100 \%$ ). NCCT images (slice thickness, $1.25 \mathrm{~mm}$ ) tended not to be stronger than the other two NCCT images (4/7; $57.14 \%$ vs. $3 / 7 ; 42.86 \%$ ), suggesting that the CAC score measured using NCCT images with a slice thickness of $1.25 \mathrm{~mm}$ was the most reliable.

Our study has some limitations. First, the number of patients was relatively small for the results to be generally accepted. In future studies, more patients should be recruited, and various CT techniques should be employed, using the most up-to-date CT scanners. Second, the clinical impact of coronary calcification on the chest CT has not been studied in this study.

\section{Conclusions}

We found that CAC quantification using NCCT scans with a wide detector, high pitch, and high temporal resolution scanning mode correlated highly with the ECG-gated 
$\mathrm{CaCT}$ scans. Chest CT images reconstructed with a slice thickness of $1.25 \mathrm{~mm}$ were more reliable than those with slice thickness of 0.625 or $2.5 \mathrm{~mm}$ for quantifying the CAC scores.

\section{Acknowledgments}

Funding: None.

\section{Footnote}

Reporting Checklist: The authors have completed the MDAR reporting checklist. Available at http://dx.doi.org/10.21037/ jtd-20-1371

Data Sharing Statement: Available at http://dx.doi. org/10.21037/jtd-20-1371

Conflicts of Interest: All authors have completed the ICMJE uniform disclosure form (available at http://dx.doi. org/10.21037/jtd-20-1371). The authors have no conflicts of interest to declare.

Ethical Statement: The authors are accountable for all aspects of the work in ensuring that questions related to the accuracy or integrity of any part of the work are appropriately investigated and resolved. This prospective study was conducted in accordance with the Declaration of Helsinki (as revised in 2013), and approved by the institutional review board of Gangnam Severance Hospital (Approval No. 3-2018-0047). Informed consent was taken from all the patients.

Open Access Statement: This is an Open Access article distributed in accordance with the Creative Commons Attribution-NonCommercial-NoDerivs 4.0 International License (CC BY-NC-ND 4.0), which permits the noncommercial replication and distribution of the article with the strict proviso that no changes or edits are made and the original work is properly cited (including links to both the formal publication through the relevant DOI and the license). See: https://creativecommons.org/licenses/by-nc-nd/4.0/.

\section{References}

1. GBD 2016 Mortality Collaborators. Global, regional, and national under-5 mortality, adult mortality, age-specific mortality, and life expectancy, 1970-2016: a systematic analysis for the Global Burden of Disease Study 2016. Lancet 2017;390:1084-150.

2. Roth GA, Huffman MD, Moran AE, et al. Global and regional patterns in cardiovascular mortality from 1990 to 2013. Circulation 2015;132:1667-78.

3. Correction to: Heart Disease and Stroke Statistics-2018 Update: A Report From the American Heart Association. Circulation 2018;137:e493.

4. Rumberger JA, Simons DB, Fitzpatrick LA, et al. Coronary artery calcium area by electron-beam computed tomography and coronary atherosclerotic plaque area. A histopathologic correlative study. Circulation 1995;92:2157-62.

5. Polonsky TS, McClelland RL, Jorgensen NW, et al. Coronary artery calcium score and risk classification for coronary heart disease prediction. JAMA 2010;303:1610-6.

6. Shemesh J, Henschke CI, Shaham D, et al. Ordinal scoring of coronary artery calcifications on low-dose CT scans of the chest is predictive of death from cardiovascular disease. Radiology 2010;257:541-8.

7. Brindle P, Emberson J, Lampe F, et al. Predictive accuracy of the Framingham coronary risk score in British men: prospective cohort study. BMJ 2003;327:1267.

8. Mettler FA Jr, Thomadsen BR, Bhargavan M, et al. Medical radiation exposure in the U.S. in 2006: preliminary results. Health Phys 2008;95:502-7.

9. Brenner DJ, Hall EJ. Computed tomography--an increasing source of radiation exposure. $\mathrm{N}$ Engl J Med 2007;357:2277-84.

10. Hecht HS, Cronin P, Blaha MJ, et al. 2016 SCCT/ STR guidelines for coronary artery calcium scoring of noncontrast noncardiac chest CT scans: A report of the Society of Cardiovascular Computed Tomography and Society of Thoracic Radiology. J Thorac Imaging 2017;32:W54-W66.

11. Tacelli N, Darchis C, Pontana F, et al. High-pitch, dualsource chest computed tomography angiography without electrocardiographic synchronization: evaluation of cardiac motion artifacts on pulmonary circulation. J Thorac Imaging 2013;28:376-82.

12. Schulz B, Jacobi V, Beeres M, et al. Quantitative analysis of motion artifacts in high-pitch dual-source computed tomography of the thorax. J Thorac Imaging 2012;27:382-6.

13. Agatston AS, Janowitz WR, Hildner FJ, et al. Quantification of coronary artery calcium using ultrafast computed tomography. J Am Coll Cardiol 1990;15:827-32.

14. Pletcher MJ, Tice JA, Pignone M, et al. Using the 
coronary artery calcium score to predict coronary heart disease events: a systematic review and meta-analysis. Arch Intern Med 2004;164:1285-92.

15. Rumberger JA, Brundage BH, Rader DJ, et al. Electron beam computed tomographic coronary calcium scanning: a review and guidelines for use in asymptomatic persons. Mayo Clin Proc 1999;74:243-52.

16. Raggi P, Callister TQ, Cooil B, et al. Identification of patients at increased risk of first unheralded acute myocardial infarction by electron-beam computed tomography. Circulation 2000;101:850-5.

17. Landis JR, Koch GG. The measurement of observer agreement for categorical data. Biometrics 1977;33:159-74.

18. Budoff MJ, Nasir K, Kinney GL, et al. Coronary artery and thoracic calcium on noncontrast thoracic CT scans: comparison of ungated and gated examinations in patients from the COPD Gene cohort. J Cardiovasc Comput Tomogr 2011;5:113-8.

19. Wu MT, Yang P, Huang YL, et al. Coronary arterial calcification on low-dose ungated MDCT for lung cancer screening: concordance study with dedicated cardiac CT. AJR Am J Roentgenol 2008;190:923-8.

20. Shemesh J, Henschke CI, Farooqi A, et al. Frequency of coronary artery calcification on low-dose computed tomography screening for lung cancer. Clin Imaging

Cite this article as: Shin JM, Kim TH, Kim JY, Park CH. Coronary artery calcium scoring on non-gated, non-contrast chest computed tomography (CT) using wide-detector, highpitch and fast gantry rotation: comparison with dedicated calcium scoring CT. J Thorac Dis 2020;12(10):5783-5793. doi: $10.21037 /$ jtd-20-1371
2006;30:181-5.

21. Hutt A, Duhamel A, Deken V, et al. Coronary calcium screening with dual-source CT: reliability of ungated, high-pitch chest CT in comparison with dedicated calcium-scoring CT. Eur Radiol 2016;26:1521-8.

22. Azour L, Kadoch MA, Ward TJ, et al. Estimation of cardiovascular risk on routine chest CT: Ordinal coronary artery calcium scoring as an accurate predictor of Agatston score ranges. J Cardiovasc Comput Tomogr 2017;11:8-15.

23. Ebersberger U, Eilot D, Goldenberg R, et al. Fully automated derivation of coronary artery calcium scores and cardiovascular risk assessment from contrast mediumenhanced coronary CT angiography studies. European Radiology 2013;23:650-7.

24. Takx RAP, de Jong PA, Leiner T, et al. Automated Coronary Artery Calcification Scoring in Non-Gated Chest CT: Agreement and Reliability. PLoS One 2014;9:e91239.

25. Xie Y, Cham MD, Henschke C, et al. Automated coronary artery calcification detection on low-dose chest CT images. Proc. SPIE 9035, Medical Imaging 2014: Computer-Aided Diagnosis, 90350F, 20 March 2014. doi: 10.1117/12.2043840.

26. Kalisz K, Buethe J, Saboo SS, et al. Artifacts at Cardiac CT: Physics and Solutions. Radiographics 2016;36:2064-83. 